\title{
Optical Design Considerations Relevant to Reflective UV Launch Gratings for Photocathode Irradiation*
}

\author{
Paul R. Bolton, \\ bolton@slac.stanford.edu, SLAC
}

\section{Introduction:}

The characteristics of photoelectron microbunches emitted from a photocathode in response to laser irradiation determine many of the incident laser pulse requirements. $\mathrm{RF}$ photocathode designs based on grazing incidence of the irradiation benefit from the removal of launch optics from the electron beamline and enhanced absorption at Brewster angles. However, this also introduces two well known complexities in the laser pulse 'launch' requirements: (i) a transverse spatial anamorphism to guarantee that the projected transverse spatial profile of the irradiation is circular (in the plane of the photocathode) and (ii) a 'time slew' or tilted amplitude front on the laser pulse that is incident on the photocathode to guarantee that the temporal (longitudinal) profiles are synchronous across the entire transverse irradiation profile in the photocathode plane. A single diffraction grating can be used to fulfill these combined requirements. This reported work focuses on grating behavior only. It does not address imaging requirements associated with relayed optical transport from the grating to the photocathode. Because the grating is a highly dispersive optical element by design, the dispersive aspects of all launch requirements are important.

\section{Basic Grating Relationships:}

Typical laser pulses (ir, visible or uv wavelengths) considered for photocathode irradiation are assumed to be of short duration, $\tau$ (several picoseconds) and with bandwidths, $\delta \lambda$ up to several nanometers. Uv irradiation is considered here. It is also assumed that the laser pulses are linearly polarized with an orientation along the ' $\mathrm{P}$ ' direction (i.e. orthogonal to the direction of the grating grooves). Figure 1 illustrates the geometry that is considered for which the grating and photocathode are separated by a center-to-center distance, $\mathrm{L}$. With respect to the grating, the incident and diffracted angles are $\alpha$ and $\beta$ respectively. The relative tilt angle, $\gamma$ is defined as the angular separation between the grating and photocathode normals. Nonzero $\gamma$ allows for some realistic nonparallelism that could exist between the grating and the photocathode in a gun design but also affords final adjustment for critical anamorphic and amplitude front tilt settings that will be discussed.

\footnotetext{
* Work supported in part by the DOE Contract DE-AC02-76SF00515.

This work was performed in support of the LCLS project at SLAC
} 
The following standard diffraction grating formula is used:

$$
\sin (\alpha)+\sin (\beta)=m \sigma \lambda
$$

The diffraction order, $m$ is $+1, \sigma$ is the groove density of the grating (in grooves per millimeter units), and $\lambda$ is the incident laser wavelength. The value of the diffraction angle, $\beta$ is positive and typically large. For chirped incident laser waveforms $\lambda \equiv \lambda(t)$ and therefore $\beta \equiv \beta(\lambda(t))$ in equation (1). The incident angle, $\alpha$, is assumed to be small (less than a few degrees) and can be either positive or negative. This is also true for the relative tilt angle, $\gamma$ which is discussed later in this report. The sign convention for angle values is chosen such that in figure 1 , the illustrated $\alpha, \beta$ and $\gamma$ angles are all positive. Let $a$ and $b$ represent the incident and diffracted beam diameters in the dispersion plane (defined here to be the full beam width at half of the maximum intensity value, FWHM where it is also assumed that the laser beam profiles are transversely uniform). The projections of these diameters, in the plane of the grating, are equal and given as:

$$
\frac{a}{\cos (\alpha)}=\frac{b}{\cos (\beta)} \equiv d
$$

Accordingly, the transverse beam size ellipticity, $\varepsilon$ is altered at the grating by diffraction from the incident value of $\frac{a}{h}$ to the diffracted value of $\frac{b}{h}$ which is then incident on the photocathode, where $h$ is the beam diameter orthogonal to the dispersion plane. In this work, we assume a circular incident profile (i.e. $a=h$ ). For normal incidence, $d=a$. The tilt of the diffracted amplitude front (as illustrated by the end points, A and B in figure 1) is defined as the longitudinal delay length, $c \delta t \equiv \delta z$ across the diffracted beam diameter. It is determined at the grating by diffraction to be:

$$
c \delta t=a \tan (\alpha)+b \tan (\beta)
$$

The time slew is then $\delta t$. 


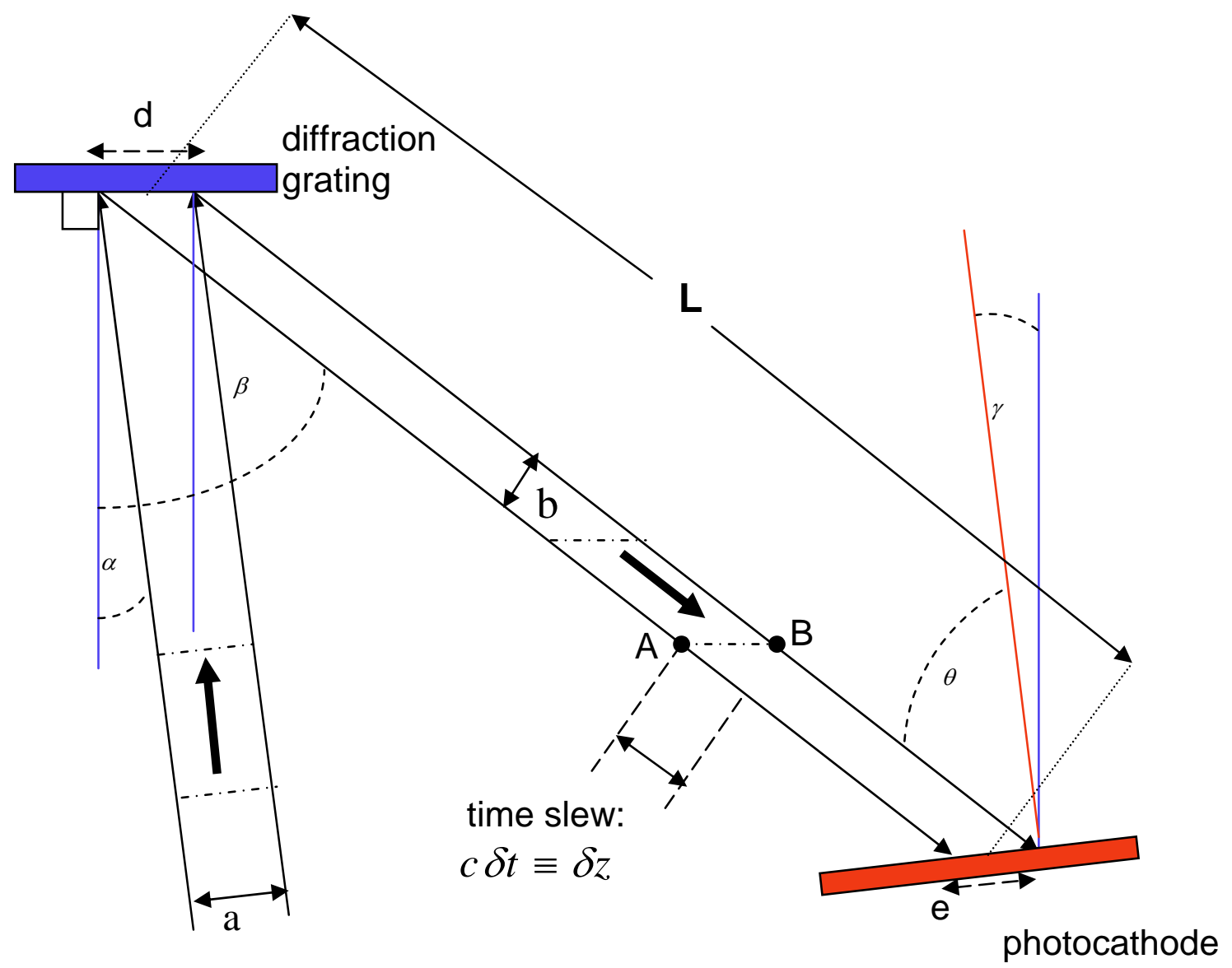

Figure 1. Combined grating-photocathode geometry.

Nominal values used in this report for beam parameters incident on the grating and diffracted beam parameters (which are then incident on the photocathode) are listed in Table 1. 
Table 1. Nominal incident and refracted beam parameter values.

Incident beam

$$
\begin{aligned}
\lambda_{o} & =255 \mathrm{~nm} \\
\sigma & =3600 \mathrm{gpm} \\
m & =+1 \\
\alpha & =0 \\
a & =2.40 \mathrm{~mm} \\
\delta \lambda & =5 \mathrm{~nm}
\end{aligned}
$$

\section{Diffracted beam}

$\beta=66.64$ degrees

$b=0.95 \mathrm{~mm}$

$\varepsilon=\frac{b}{a}=0.40$

$c \delta t=2.20 \mathrm{~mm}$

$\delta t=7.34$ psec

$\gamma_{a}=0$

$\gamma_{t}=0$

\section{Special Corrective Relative Tilt Angles:}

\subsection{Anamorphic Correction:}

For a nonzero relative tilt, $\gamma$ the projected beam diameter, $e$ in the dispersion plane and in the photocathode plane is given as:

$$
\frac{b}{\cos (\beta-\gamma)} \equiv e
$$

It can be greater or less than that in the grating plane, $d$ for negative or positive $\gamma$ values respectively. To establish a projected circular irradiation profile in the photocathode plane requires a unique relative tilt angle, $\gamma_{a}$ that corrects for the anamorphism and is determined from the incident angle, $\alpha$ according to:

$$
\begin{aligned}
& \cos \left(\beta-\gamma_{a}\right) \equiv \frac{\cos (\beta)}{\cos (\alpha)} \equiv \frac{b}{a} ; \gamma_{a} \geq 0 \\
& \tan \left(\gamma_{a}\right)=\frac{\left(1-\cos (\alpha) \cos \left(\gamma_{a}\right)\right)}{\left(\tan (\beta) \cos (\alpha) \cos \left(\gamma_{a}\right)\right)}
\end{aligned}
$$


Because the relative tilt angles are typically small, we can approximate $\gamma_{a}$ as follows:

$$
\tan \left(\gamma_{a}\right) \approx \sin \left(\gamma_{a}\right) \approx \frac{(1-\cos (\alpha))}{\tan (\beta) \cos (\alpha)}
$$

As expected, we require the grating and photocathode planes to be parallel (i.e. $\gamma_{a}=0$ ) for the special case of normal incidence at the grating $(\alpha=0)$. In general, the corrective relative tilt angle, $\gamma_{a}$ must be nonnegative and, at the photocathode, recovers the original circular beam of diameter, $a$ that is incident on the grating. It is treated as an anamorphic correction for nonzero $\alpha$.

\subsection{Amplitude Front Tilt Correction:}

For irradiation that is incident on the photocathode at an angle, $\theta$ the 'required' amplitude front tilt (with respect to the photocathode plane) that establishes synchronous irradiation across this plane depends only on the beam size, $b$ and $\theta$ according to:

$$
\begin{aligned}
& c \delta t \equiv b \tan (\theta) \\
& \text { where } \theta \equiv \beta-\gamma
\end{aligned}
$$

As with the anamorphic requirements discussed above, for the case of amplitude front tilt, the grating and photocathode planes must be parallel for normal incidence at the grating. If $\alpha$ is nonzero, the grating and photocathode planes that guarantee synchronous irradiation across the photocathode are tilted relative to one another by a different corrective angle, $\gamma_{t}$ which can be determined according to:

$$
c \delta t=b \tan \left(\beta-\gamma_{t}\right)=a \tan (\alpha)+b \tan (\beta)
$$

Therefore, $\gamma_{t}$ can be expressed in terms of the grating angles and the amplitude front tilt as follows: 


$$
\tan \left(\gamma_{t}\right) \equiv \frac{-a \tan (\alpha)}{(b+(c \delta t) \tan (\beta))}
$$

Angles, $\alpha$ and $\gamma_{t}$ have opposite signs for positive $\beta$.

For a single relative tilt angle, $\gamma_{a t}$ to act as a combined correction for both anamorphism and the amplitude front tilt we would require:

$$
\alpha \leq 0 \text { and } \gamma_{a}=\gamma_{t} \equiv \gamma_{a t} \geq 0
$$

\subsection{Spectral Dispersion:}

A single laser pulse is characterized by some nonzero bandwidth, $\delta \lambda$. Spectral dispersion from the grating introduces a bandwidth limited range of values for $b, \beta, c \delta$, and $\delta t$. The sensitivity of these parameters to wavelength can then be expressed in terms of the following derivatives with respect to wavelength:

$$
\begin{aligned}
& \frac{d \beta}{d \lambda}=\frac{m \sigma}{\cos (\beta)} \\
& \frac{d^{2} \beta}{d \lambda^{2}}=\left(\frac{d \beta}{d \lambda}\right)^{2} \tan (\beta)
\end{aligned}
$$

$$
\begin{aligned}
& \frac{d b}{d \lambda}=-\left(\frac{m a \sigma}{\cos (\alpha)}\right) \tan (\beta)=-b\left(\frac{d \beta}{d \lambda}\right) \tan (\beta) \\
& \frac{d(c \delta t)}{d \lambda}=\frac{m a \sigma}{\cos (\alpha)}=b\left(\frac{d \beta}{d \lambda}\right)
\end{aligned}
$$


Note that the dispersions, $\frac{d b}{d \lambda}, \frac{d(c \delta t)}{d \lambda}$, and $\frac{d^{2} \beta}{d \lambda^{2}}$ can be expressed in terms of their scaling with $\frac{d \beta}{d \lambda}$. It is assumed that there is no significant dispersion for incident beam diameter, $a$ and angle, $\alpha$. Note also that for positive $\beta$, first and second order $\beta$ derivatives have the same sign. For positive order, $m$ the time slew derivative is positive. The significance of spectral dispersion for the nominal input values of Table 1 is indicated in Table 2 where wavelength derivatives are listed for nominal parameter values.

Table 2. Nominal wavelength derivatives at $255 \mathrm{~nm}$.

\section{Nominal wavelength derivatives}

$$
\begin{aligned}
\frac{d b}{d \lambda} & =-2.00 \times 10^{-2} \mathrm{~mm} / \mathrm{nm} \\
\frac{d \beta}{d \lambda} & =9.078 \mathrm{mrad} / \mathrm{nm} \\
\frac{d^{2} \beta}{d \lambda^{2}} & =0.191 \mathrm{mrad} /\left(\mathrm{nm}^{2}\right) \\
\frac{d(c \delta t)}{d \lambda} & =8.64 \times 10^{-3} \mathrm{~mm} / \mathrm{nm} \\
\frac{d(\delta t)}{d \lambda} & =28.83 \mathrm{fsec} / \mathrm{nm} \\
\frac{d \gamma_{a}}{d \lambda} & =0.0 \mathrm{mrad} / \mathrm{nm} \\
\frac{d \gamma_{t}}{d \lambda} & =0.0 \mathrm{mrad} / \mathrm{nm}
\end{aligned}
$$




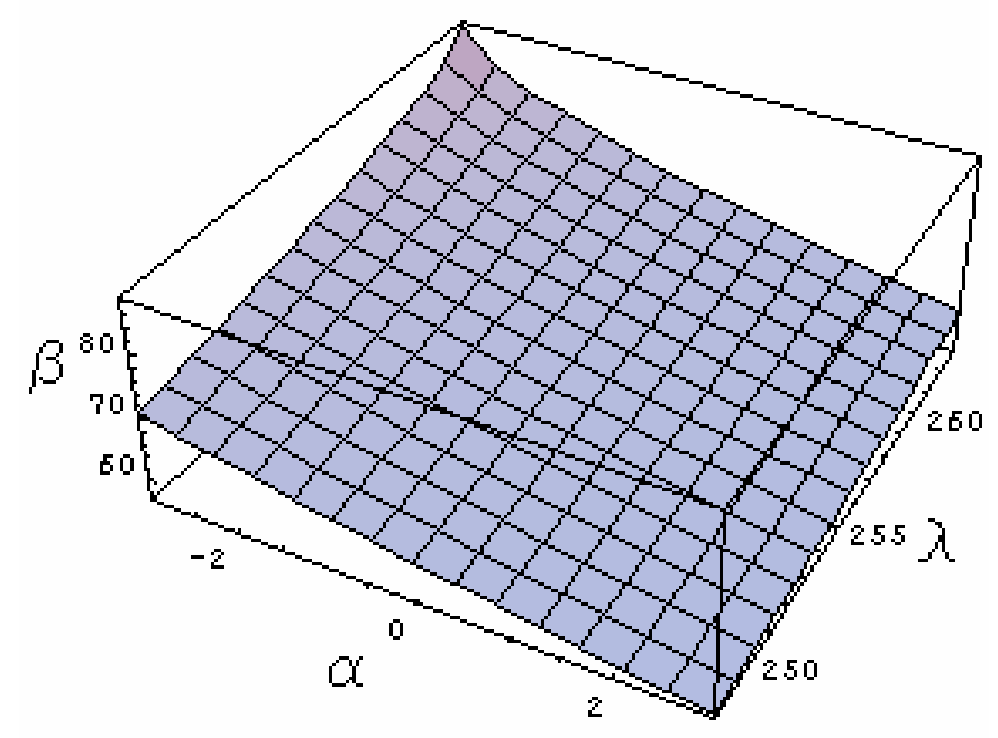

Figure 2. Variation of the diffractive angle (degrees) with incidence angle (degrees) and wavelength (nm) for a grating groove density of 3600 grooves per mm.

Figures 2 to 4 illustrate the incident angle, $\alpha$ and wavelength, $\lambda$ dependences of parameters $\beta, b$, and $\delta t$ that are established by the grating alone. It should be noted here that the variations of $\alpha$ and $\lambda$ are to be interpreted differently. The bandwidth represents a range of wavelength values that applies to any individual laser pulse, whereas the variation of incident angle is interpreted as change in a specific single value that applies to any laser pulse (i.e. the collimated case). This is also the case for the incident angle and wavelength dependences of the two special corrective tilt angles that are shown in figures 5 and 6 . These two corrective angles are also completely determined by the incident and diffracted parameters at the grating.

Figure 2 illustrates this for the diffracted angle, $\beta$. For normal incidence, the first (second) order variation, $\delta \beta$ over a $5 \mathrm{~nm}$ wavelength interval is about $2.6(0.26)$ degrees as confirmed in equation (11). For a fixed wavelength, the variation with $\alpha$ reveals comparable sensitivity but with opposite sign. 


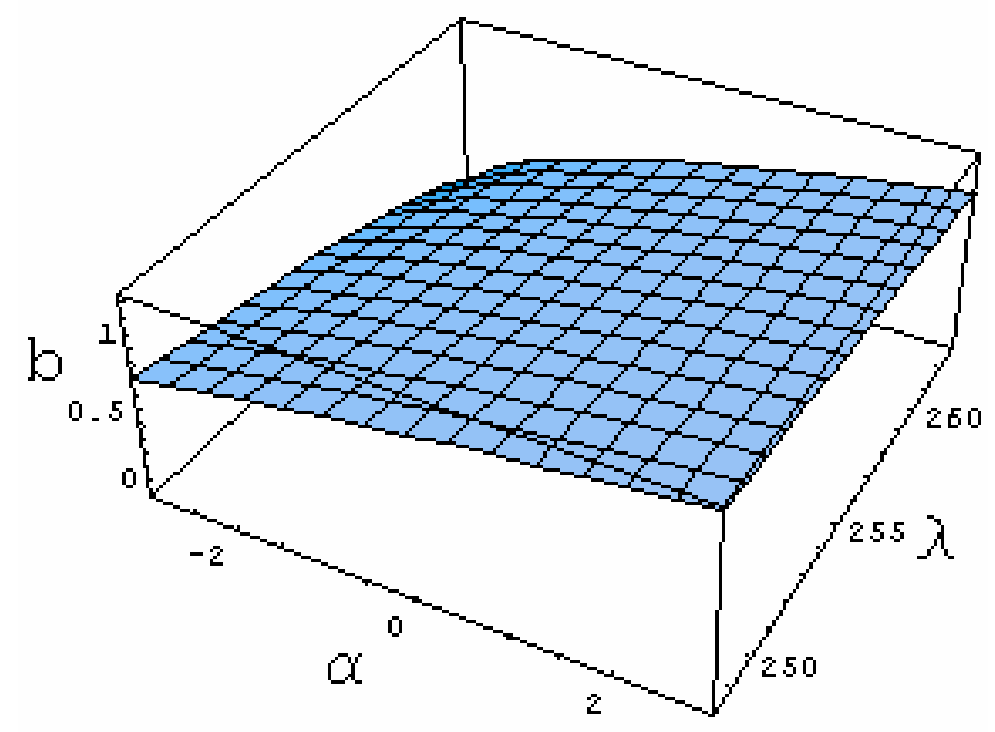

Figure 3. Variation of the diffracted beam diameter ( $\mathrm{mm}$ ) with incident angle (degrees) and wavelength (nm) for a grating groove density of 3600 grooves per $\mathrm{mm}$ and an incident beam diameter of $2.4 \mathrm{~mm}$.

Similarly, figure 3 illustrates the dependence of the diffracted beam diameter, $b$ with incident angle and wavelength. Variations in the beam diameter increase with reduced incident angle and with increased wavelength. At normal incidence, a $5 \mathrm{~nm}$ bandwidth corresponds to a diffracted beam diameter variation of approximately 100 microns, consistent with equation (11). For a given wavelength, the variation of $b$ with incident angle is larger. For example, at $255 \mathrm{~nm}$, a 3 degree change in incident angle results in a diffracted beam diameter variation of about 300 microns.

Figure 4 illustrates the time slew, $\delta t$ variation with incident angle and wavelength where it is observed that a linear wavelength dependence dominates. For normal incidence and a $5 \mathrm{~nm}$ wavelength interval the time slew variation is at the 144 femtosecond level, consistent with equation (11). 


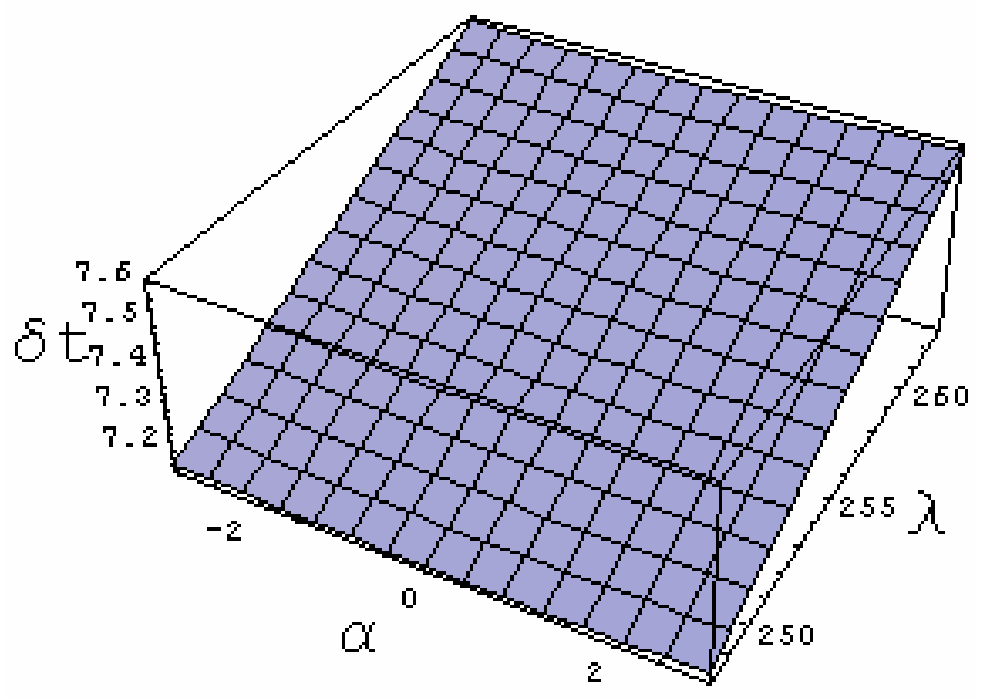

Figure 4. Variation of time slew (picoseconds) with incident angle (degrees) and wavelength (nm) for a grating groove density of 3600 grooves per $\mathrm{mm}$ and an incident beam diameter of $2.4 \mathrm{~mm}$.

In the absence of any imaging between the grating and photocathode surfaces, the increase in the projected beam diameter in the photocathode plane (beyond the value, $d$ ) that is attributed to spectral dispersion of the diffracted angle, $\beta$ is estimated to be $\operatorname{Lm} \sigma(\delta \lambda) \approx 0.018 L$ for the nominal $5 \mathrm{~nm}$ bandwidth. This increase is comparable to the nominal value of $d$ (which is $a=2.4 \mathrm{~mm}$ ) for $L \approx 133 \mathrm{~mm}$. Because practical values of $L$ can be larger than $133 \mathrm{~mm}$, this increase is typically much larger than the contribution from the spectral dispersion of $b$ (which is only about 250 microns in the photocathode plane for the $5 \mathrm{~nm}$ bandwidth).

The spectral dispersion of the two corrective relative tilt angles, $\gamma_{a}$ and $\gamma_{t}$ is also expressed in terms of their wavelength derivatives as follows: 


$$
\begin{aligned}
& \frac{d \gamma_{a}}{d \lambda}=\frac{1}{\sin (\beta) \cos (\beta)}\left(\frac{\cos (\alpha) \cos ^{2}\left(\gamma_{a}\right)-\cos \left(\gamma_{a}\right)}{\cos (\alpha) \tan (\beta)-\sin \left(\gamma_{a}\right)}\right)\left(\frac{d \beta}{d \lambda}\right) \\
& \text { and } \\
& \frac{d \gamma_{t}}{d \lambda} \equiv \frac{-\cos \left(\gamma_{t}\right) \sin \left(\gamma_{t}\right)}{(b+(c \delta t) \tan (\beta))}\left\{\frac{m \sigma(c \delta t)}{\cos ^{3}(\beta)}\right\}=\frac{-\cos \left(\gamma_{t}\right) \sin \left(\gamma_{t}\right)}{(b+(c \delta t) \tan (\beta))}\left(\frac{c \delta t}{\cos ^{2}(\beta)}\right)\left(\frac{d \beta}{d \lambda}\right)
\end{aligned}
$$

These derivatives also scale with $\frac{d \beta}{d \lambda}$. The $\gamma_{a}$ derivative is negative for positive order, $m$ and positive $\beta$. The $\gamma_{t}$ derivative is positive for positive order, $m$ and negative $\gamma_{t}$. They are both equal to zero for the nominal case of normal incidence at the grating.

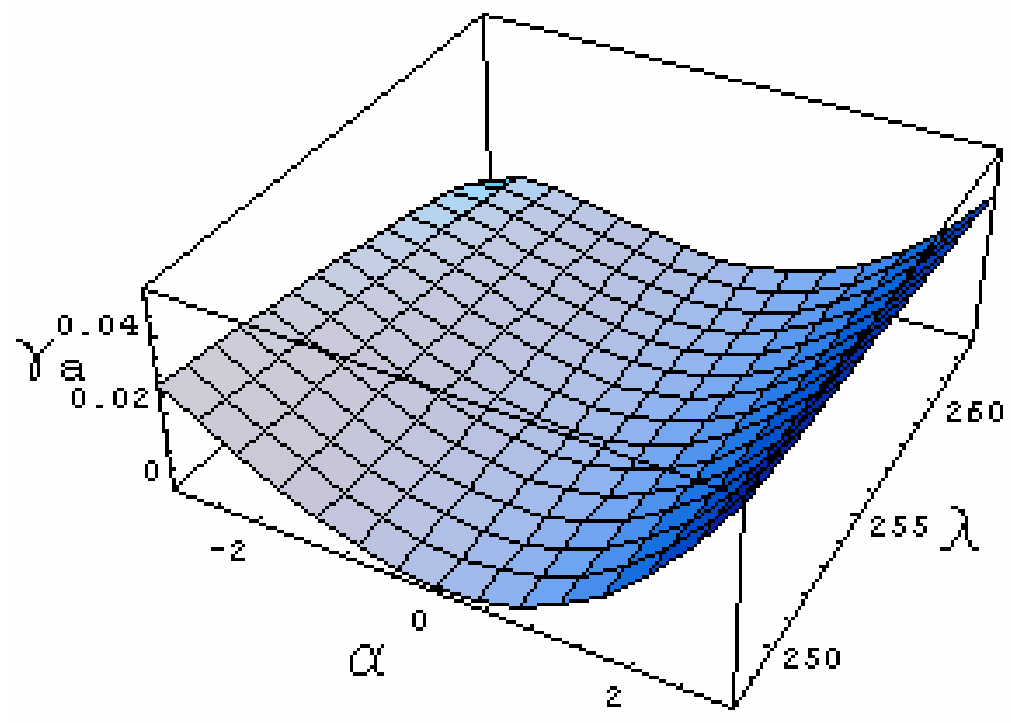

Figure 5. Variation of the relative tilt angle for correction of anamorphism (degrees) with incident angle (degrees) and wavelength (nm) for a grating groove density of 3600 grooves per $\mathrm{mm}$ and an incident beam diameter of $2.4 \mathrm{~mm}$. 
Figure 5 reveals the dependence of $\gamma_{a}$ on incident angle and wavelength where the constraint to nonnegative values is clear. The sensitivity to incident angle variation is much stronger than that for wavelength variation. Even so, for a $255 \mathrm{~nm}$ wavelength and a 3 degree change in incident angle, $\gamma_{a}$ only varies no more than about 0.04 degrees.

Similar results are shown in figure 6 for the variation of $\gamma_{t}$ where, again, the incident angle dependence dominates. However, the variation of $\gamma_{t}$ with incident angle is significantly stronger (almost two orders of magnitude) than that for $\gamma_{a}$. For a $255 \mathrm{~nm}$ wavelength, the $\gamma_{t}$ variation imposed by a 3 degree change in incident angle is at the 1.5 degree level.

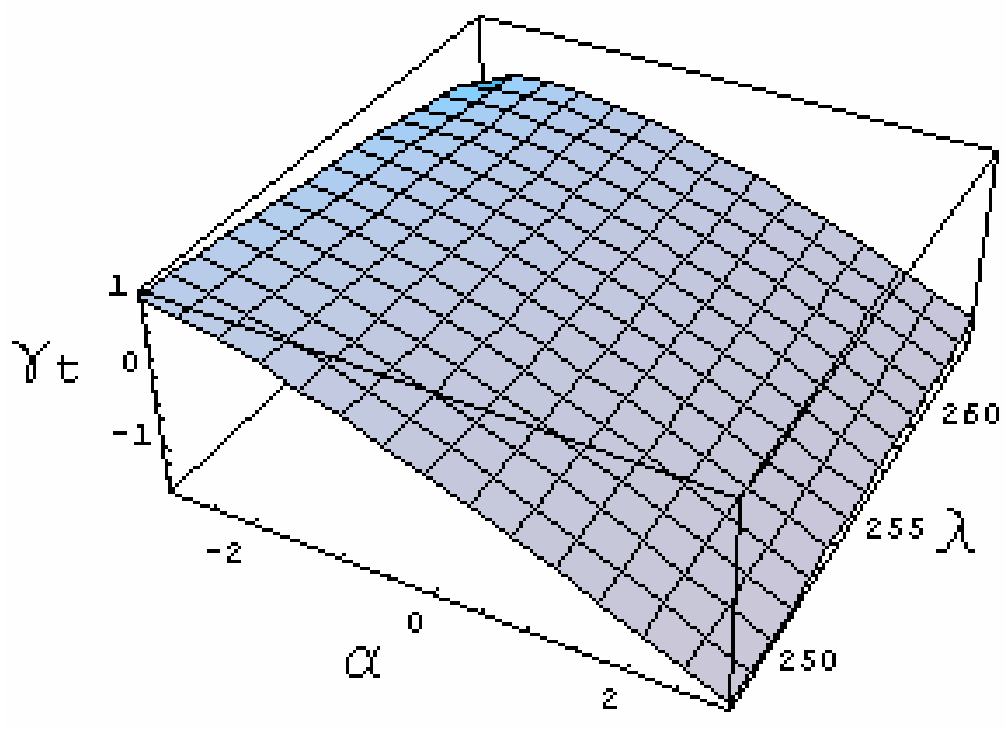

Figure 6. Variation of the relative tilt angle for correction of amplitude front tilt (degrees) with incident angle (degrees) and wavelength (nm) for a grating groove density of 3600 grooves per $\mathrm{mm}$ and an incident beam diameter of $2.4 \mathrm{~mm}$.

\section{Summary:}

For a fixed incident angle, $\alpha$ an amplitude front tilt correction angle, $\gamma_{t}$ exists as determined from equation (9) and is of opposite sign. With respect to amplitude front tilt, one can then consider the regions of under and over corrective tilt to be those for which $\gamma<\gamma_{t}$ and $\gamma>\gamma_{t}$ respectively. For this same incident angle (regardless of sign) the 
anamorphic correction angle, $\gamma_{a}$ must be positive and given by equations (5) and (6). Similarly, with respect to the anamorphism, one can consider the regions of under and over corrective tilt to be those for which $\gamma<\gamma_{a}$ and $\gamma>\gamma_{a}$ respectively. These two special relative tilts correct (or compensate) for a given incident angle at the grating. Both corrections are equal and zero for normal incidence at the grating making the grating and photocathode parallel. For grating incidence that is not normal, the grating and photocathode are not parallel in the optimum configuration.

One can alternatively consider the grating incidence angle as the correction for a relative tilt, $\gamma$ with the same constraints and using the same specified relationships in equations (5), (6) and (9). However, for this complementary case, there exists no $\alpha$ correction for anamorphism when the relative tilt, $\gamma$ is negative.

Given the choice, one should preferentially correct for the amplitude front tilt. This is because (i) there is no absolute sign constraint and one can choose either an $\alpha$ or $\gamma$ correction and (ii) where correction for anamorphism is necessary it is possible to adjust projected irradiance beam profiles by other means such as imaging an appropriately rotated hard edge aperture that is placed between the grating and the photocathode. These findings are intended to assist with the design of optical transport systems for photocathode irradiation and with associated gun design issues. 